\title{
On the possibility of detecting extrasolar planets' atmospheres with the Rossiter-McLaughlin effect
}

\author{
S. Dreizler ${ }^{1}$, A. Reiners ${ }^{1}$, D. Homeier ${ }^{1}$, and M. Noll ${ }^{1,2}$ \\ 1 Institut für Astrophysik, Georg-August-Universität Göttingen, Friedrich-Hund-Platz 1, 37077 Göttingen, Germany \\ e-mail: dreizler@astro.physik.uni-goettingen.de \\ 2 Gymnasium am Bötschenberg, Am Bötschenberg 11, 38350 Helmstedt, Germany
}

Received 23 July 2008 / Accepted 23 February 2009

\section{ABSTRACT}

\begin{abstract}
Context. The detection of extrasolar planets' atmospheres requires very demanding observations. For planets that cannot be spatially separated from their host stars, i.e. the vast majority of planets, the transiting planets are the only ones to allow their atmospheres to be probed. This is possible from transmission spectroscopy or from measurements taken during the secondary eclipse. An alternative is to measure of the Rossiter-McLaughlin effect, which is sensitive to the size of the planetary radius. Since the radius is wavelengthdependent due to contributions of strong planetary absorption lines, this opens a path toward also probing planetary atmospheres with ground-based high-resolution spectroscopy.

Aims. The major goal of our numerical simulations is to provide a reliable estimate of the amplitude of the wavelength-dependent Rossiter-McLaughlin effect.

Methods. Our numerical simulations provide detailed phase-resolved synthetic spectra modeling the partly eclipsed stellar surface during the transit. With these spectra we can obtain Rossiter-McLaughlin curves for different wavelength regions and for a wavelengthdependent planetary radius. Curves from regions with high and low contributions of absorption lines within the planetary atmosphere can be compared. Observable quantities are derived from these differential effects.

Results. We applied our simulations to HD 209458. Our numerical simulations show that a detailed treatment of the limb-darkening for the synthetic spectra is important for a precise analysis. Compared to a parameterized limb-darkening law, systematic errors of $6 \mathrm{~m} \mathrm{~s}^{-1}$ occur. The wavelength dependency of the planetary atmospheres over the NaD-doublet produces a differential effect in the Rossiter-McLaughlin curve of $1.5 \mathrm{~m} \mathrm{~s}^{-1}$ for a star with a rotation velocity of $4.5 \mathrm{~km} \mathrm{~s}^{-1}$, which increases to $4 \mathrm{~m} \mathrm{~s}^{-1}$ for twice the rotation velocity.

Conclusions. As a tool for probing planetary atmospheres the Rossiter-McLaughlin effect requires phase-resolved, high signal-tonoise, high-resolution spectra taken with a stabilized spectrograph in order to obtain reliable results for slowly rotating $\left(<10 \mathrm{~m} \mathrm{~s}^{-1}\right)$ planet host stars. Stars with spectral type earlier than about F5 are a bit less demanding since the typically higher rotation velocity increases the amplitude of the effect to about $15 \mathrm{~m} \mathrm{~s}^{-1}$ for a star with $v \sin i=25 \mathrm{~km} \mathrm{~s}^{-1}$.
\end{abstract}

Key words. planetary systems - techniques: radial velocities - line: profiles - stars: rotation - stars: individual: HD 209458

\section{Introduction}

Within the two recent decades, the search for extrasolar planets has been mainly driven by the success of the radial velocity (RV) method. A large fraction of our knowledge about the physical properties relies, however, on analyses of transiting planets, because more information can be obtained from various techniques for transiting planets: the radius and the orbital inclination from the light curve analysis together with the mass function from $\mathrm{RV}$-measurements provide the mean planetary density. This is an important constraint for the structure and evolution of the planet. The atmosphere can be probed by means of transmission spectroscopy during transits (Charbonneau et al. 2002; Vidal-Madjar et al. 2003, 2004; Barman 2007; Tinetti et al. 2007; Ehrenreich et al. 2008; Lecavelier Des Etangs et al. 2008a,b; Pont et al. 2008; Redfield et al. 2008; Sing et al. 2008a,b; Snellen et al. 2008; Swain et al. 2008b), and the albedo and thermal emission can be determined from the secondary eclipse with infrared photometry (Charbonneau et al. 2005; Deming et al. 2005, 2006, 2007; Demory et al. 2007; Grillmair et al. 2007; Harrington et al. 2007; Richardson et al. 2007; Swain et al. 2008a).
From eclipsing binary systems it has been known for decades that the radial velocity curve shows a characteristic feature during eclipses (Schlesinger 1910; Rossiter 1924; McLaughlin 1924). This Rossiter-McLaughlin effect (RME) can be explained by partially covering the eclipsed star. Out of eclipse, the spectral lines will be symmetric since all surface elements of the (rotating) star contribute equally, thus blue- and red-shifted regions of the surface are balanced. This changes during the eclipse depending on the path of the occulting body with respect to the eclipsed star, resulting in asymmetric lines due to imbalanced Doppler-shifted contributions. Depending on the spectral resolution of the spectrograph and the rotational velocity of the star, this can be detected either as pseudo RV variation (slow rotation or low resolution) or directly as line distortion moving over the spectral lines during the eclipse. The RME has been shown to be a powerful tool for transiting planets as well. The method has been applied for measuring of the spin-orbit alignment for eleven of the transiting planets (Bundy \& Marcy 2000; Queloz et al. 2000; Winn et al. 2005, 2006, 2007; Narita et al. 2007; Wolf et al. 2007; Bouchy et al. 2008; Cochran et al. 2008; Hébrard et al. 2008; Johnson et al. 2008; Joshi et al. 2008; Loeillet et al. 2008; Narita et al. 2008). With the fast-growing number of bright 
host stars of transiting planets, either from ground-based widefield surveys or from space missions, this measurement turns into a standard tool for investigating the properties of extrasolar planets.

The RME has also been proposed as tool for probing planetary atmospheres with ground-based observations (Snellen 2004). Since transmission spectroscopy, as well as albedo measurements, mainly relies on the high precision obtainable only from space-based measurements (see Redfield et al. 2008; Snellen et al. 2008 as exceptions), ground-based access is an attractive alternative. The RME therefore is a complementing rather than a competing method. The method detecting planetary atmospheres with the RME is based on the radius of an extrasolar planet depending on wavelength. This is caused, on the one hand, by the atmosphere of the planet showing variations in optical depth with respect to the wavelength (Barman 2007; Fortney et al. 2008). The planet will appear slightly larger in optically thick spectral lines compared to wavelength regions where the atmosphere is transparent. On the other hand, the amplitude of the RME depends on the radius of the occulting body. A wavelength-resolved measurement of that effect therefore provides access to the atmospheric properties of the transiting planet.

While the measurements by Snellen (2004) were at the limit of the available data, it is the aim of this paper to provide detailed modeling of the RME in order to provide a reliable analysis tool for such demanding measurements. We therefore describe the underlying model in Sect. 2, present test calculation for HD 209458 in Sect. 3, and summarize the results in Sect. 4.

\section{Modeling the Rossiter-McLaughlin effect}

Recently, there have been two papers on analytical expressions for the RME, expressing the RV-shift in terms of orbital parameters and radius of the planet, orbital orientation relative to the rotational axis, stellar rotation velocity, radius, and limbdarkening (Ohta et al. 2005; Giménez 2006) and making use of earlier analytical work (Petrie 1938; Hosokawa 1953; Kopal 1959). While the analytical models are well-suited as fast and direct method, Winn et al. (2005) argue that the first moment of the spectral lines, i.e. the shift in center of gravity in wavelength space as a result of the analytical formula, might not be identical to the value measured from observations. In that case, the optimal match between a template and the observed spectrum is sought.

The alternative approach is therefore a finite element model of the star by adding contributions from all surface elements to a synthetic spectrum that can then be treated with the same analysis tools as the observations, e.g. as used by Queloz et al. (2000) and Winn et al. (2005). While computationally more demanding, this approach allows deviation from simplifications needed in the analytical models allowing for a more precise modeling of the RME. In the following paragraphs the modeling of the wavelength-dependent RME will be described.

\subsection{The star}

The assumption of a spherical primary star is well-suited to the case of a planetary secondary since any deviations from spherical symmetry of the primary due to tidal interaction is negligible. Spherical symmetry also requires a slow rotation of the primary. For late type stars, this assumption is justified. It is, however, challenged when the rotation velocity becomes significantly higher than in the Sun. To prepare for such cases, we make use of the program BRUCE, originally written by Townsend (1997) to model stellar pulsations in rotating stars. A deformation of the stellar surface is calculated as perturbation providing variations in the effective temperature and surface gravity over the surface. As input, the stellar mass, the polar radius and effective temperature, the equatorial velocity, a velocity amplitude due to pulsation, and the inclination $i$ of the rotation axis (assumed to be constant) have to be specified. In our application, we set the pulsational velocity to zero (this could, however, be included for investigating the contribution of stellar pulsation to RV noise). In an output file, BRUCE provides effective temperature, surface gravity, and projected radial velocity, as well as the cosine of the angle between the normal direction of the surface element and the line of sight of the observer $(\mu)$ for the required number of surface elements (typically 50000 in our test calculations).

\subsection{Synthetic spectrum of the host star}

In previous modeling authors have used template spectra, e.g. the solar spectrum, or mean intensities from model atmospheres as input spectra. In both cases, a limb-darkening law (e.g. Claret $2000,2003,2004)$ has to be applied. For a detailed wavelengthdependent modeling of the RME, the available broad-band limbdarkening coefficients are not sufficient; instead, this would require determining the limb-darkening coefficients from model atmospheres adapted to the spectral resolution of the observations. To avoid errors due to the parameterization of the limbdarkening law, specific intensities for all surface elements of the star can be used directly for a synthetic spectrum.

The input spectra were synthesized using specific intensities (energy emitted in a given direction $\boldsymbol{n}$ per time, unit area, frequency, and solid angle interval) from a grid of PHOENIX models. The grid covers the main sequence in steps of $200 \mathrm{~K}$ for the effective temperature and with two surface gravities $(\log g=4$ and $\log g=4.5$ ). The models have been calculated for spherically symmetric atmospheres under the assumption of local thermodynamic equilibrium (Hauschildt \& Baron 1999; Hauschildt et al. 1999). The general setup is described in Brott \& Hauschildt (2005); however, we are adopting the revised solar abundances of Asplund et al. (2005) to calculate the chemical composition (for solar metallicity in the present case, but available for other metallicities as well). Specific intensity spectra for each model computed at a resolution of $0.01 \AA$ for different values of the angle $\theta$ between the normal direction of the atmosphere and the direction of the line of sight $(\mu=\cos \theta)$. Nineteen intensities per wavelength point are extracted from all core rays and a subset of the tangent rays to sample the range $0<\mu<1$ evenly. Thus while our model treats the global geometry of the stellar surface exactly including rotational flattening, it is locally approximated in spherical geometry, while interpolating to the exact effective temperature and gravitational acceleration for each atmospheric segment. Since the deformation and the total curvature are very small for dwarf stars, these approximations lead to negligible errors. The spectrum is then synthesized by linear interpolation within the available grid of intensities on a logarithmical equidistant wavelength grid of the required spectral resolution and shifting to the required Doppler velocity. For comparison, we can also calculate intensities from the disk-averaged spectrum by applying a linear limb-darkening law. The undisturbed spectrum of the planet host star is now obtained and can be used as template for cross correlations to determine the RME, and it serves as reference spectrum for the next step. 


\subsection{The planet}

The RME is dependent on the size of the planet, as well as on its orbital parameters. Since transiting planets are typically close in, the assumption of a circular orbit is justified; however, eccentric orbits with eccentricities above 0.1 have been detected in four transiting planets: GJ 436b, ecc $=0.15$ Deming et al. (2007), $\mathrm{XO}-3 \mathrm{~b}$, ecc $=0.26$ Johns-Krull et al. (2008), HD 147506b, ec $=0.5163$ Bakos et al. (2007), HD 17156b, ecc $=0.6753$ Fischer et al. (2007). We therefore specify the orbital separation and period. An extension towards eccentric orbits would be possible in case this is needed. The orientation of the planetary orbit with respect to the line of sight towards the observer also has to be specified since the Doppler shifts due to stellar rotation breaks the symmetry in this case. The orientation of the orbital rotation axis relative to the line of sight needs two additional parameters, the inclination $i$ and the tilt of the orbit in the plane perpendicular to the line of sight $\lambda$.

Synthetic spectra can now be calculated. We specify an equidistant phase sampling (starting shortly before the first contact and ending shortly after the fourth contact), the radius of the planet and the angles $i$ and $\lambda$. For each orbital phase, the elements on the stellar surface that are eclipsed are identified and their spectra interpolated as in the previous step. Their contribution is then subtracted from the reference spectrum resulting in the synthetic spectrum for the given orbital phase. This procedure can be repeated for different planetary parameters.

\subsection{Wavelength-dependent planetary radius}

From radiative transport calculations, Barman (2007) deduce a wavelength dependency of the planetary radius as the result of the varying optical depth of the planetary atmosphere (see Fig. 1 for an adopted version of his Fig. 1). From his results a variation on the order of $5 \%$ can be expected, with a prominent peak e.g. at the resonance lines of sodium. Predictions of Fortney et al. (2008) indicate a variation between 2\% and 5\% depending on the class of irradiated atmosphere. To take the wavelength-dependent radius into account, we calculated two sets of synthetic spectra $\left(F_{\text {up }}, F_{\text {low }}\right)$, one with the upper limit and one with the lower limit of the radius $\left(r_{\text {up }}, r_{\text {low }}\right)$. For each wavelength point, we then interpolate the two synthetic spectra for the given wavelength point to the value according to the prediction of Barman (2007), i.e. $F_{\text {int }, \lambda}=F_{\text {low }}+\left(r_{\text {int }, \lambda}-r_{\text {low }}\right) \cdot\left(F_{\text {up }}-\right.$ $\left.F_{\text {low }}\right) /\left(r_{\text {up }}-r_{\text {low }}\right)$. It should be noted that we are not restricted to these prediction but can accommodate any given wavelengthdependent radius, e.g. based on observations of transmission spectra (Sing et al. 2008a). The application of a linear interpolation is justified as can be seen in Fig. 2. In the upper panel we display the RME for the lower and upper limits of the predicted wavelength-dependent radius as well as the RME for an intermediate value, one calculated directly as described in the previous step, the other one interpolated between the two limiting cases. The lower panel shows the difference between the two, which are close to $\mathrm{cm} \mathrm{s}^{-1}$ and therefore certainly below current detection limits.

\section{Results: Test case HD 209458}

In the following, we present test calculations of the RME and transit light curves for HD 209458. The stellar and planetary parameters are adopted from Nordström et al. (2004) and Brown et al. (2001), namely we use $R_{* \text {,polar }}=1.15 R_{\odot}, M_{*}=1.05 M_{\odot}$, $v \sin i=4.5 \mathrm{~km} \mathrm{~s}^{-1}, T_{\text {eff,polar }}=6000 \mathrm{~K}$, and an inclination of

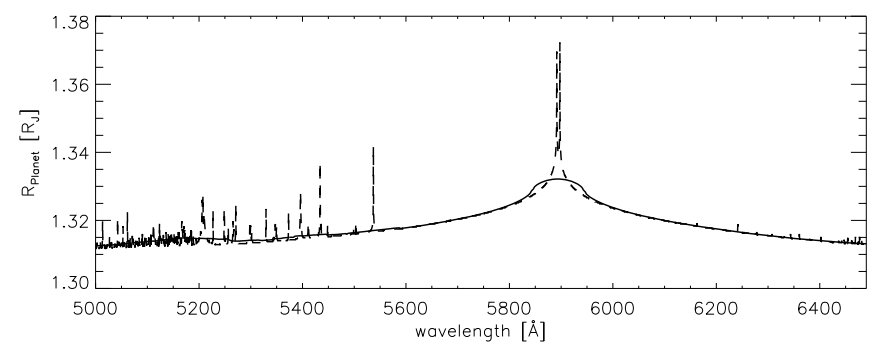

Fig. 1. Wavelength-dependent planetary radius of HD 209458b (Barman 2007) (dashed line) and smoothed over $100 \AA$ A bins (full line).

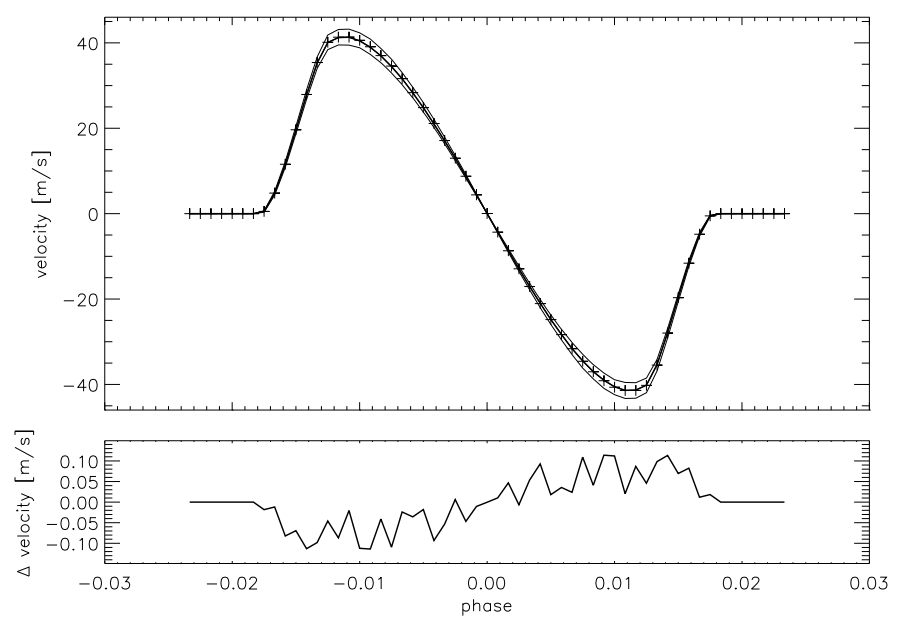

Fig. 2. Rossiter-McLaughlin effect for HD 209458 (orbital motion subtracted): top panel: planetary radius $R_{\mathrm{P}}=1.37 R_{\text {Jup }}$ and $R_{\mathrm{P}}=1.31 R_{\text {Jup }}$ (thin lines, full simulation), $R_{\mathrm{P}}=1.34 R_{\mathrm{Jup}}$ (thick line, full simulation), and $R_{\mathrm{P}}=1.34 R_{\mathrm{Jup}}$ (++++, interpolation procedure using $R_{\mathrm{P}}=1.37 R_{\mathrm{Jup}}$ and $\left.R_{\mathrm{P}}=1.31 R_{\mathrm{Jup}}\right)$. Lower panel: difference between the full simulation and interpolation procedure for $R_{\mathrm{P}}=1.34 R_{\text {Jup }}$. See text for details.

the rotational axis of $90^{\circ}$, an orbital separation of $0.047 \mathrm{AU}$, an orbital inclination of $86.6^{\circ}$, and no tilt of the orbit in the plane perpendicular to the line of sight. The planetary radius is treated wavelength-dependent (Fig. 1), as described in the previous section using the predictions of Barman (2007).

\subsection{The effect of limb-darkening}

The form of the RME is not only dependent on the stellar and planetary parameters, but also sensitive to the treatment of limbdarkening. As described in Sect. 2, our spectral synthesis relies on the co-addition of specific intensities for each surface element, interpolated to the actual value of the angle between the normal of the element and the line of sight. In Figs. 3 and 4, we compare the averaged RME from a reference region (5000-5200 A) from our full simulation including specific intensities to two versions of simulations using the flux and a linear limb-darkening law $I_{\mu, \lambda}=1+(\mu-1) u_{\lambda}$ for the spectrum synthesis. In the first case we use a constant coefficient of $u_{\lambda}=u=0.58$ used by Snellen (2004), and in the second a polynomial fit of second order to the wavelength-dependent linear limb-darkening coefficient (Fig. 5).

The deviation between the simplified limb-darkening approach and the detailed simulation using specific intensities is most evident when the planet covers the stellar limb, i.e. before the second and after the third contact. Here, the differences between the limb-darkening law and the actual $\mu$-dependence 


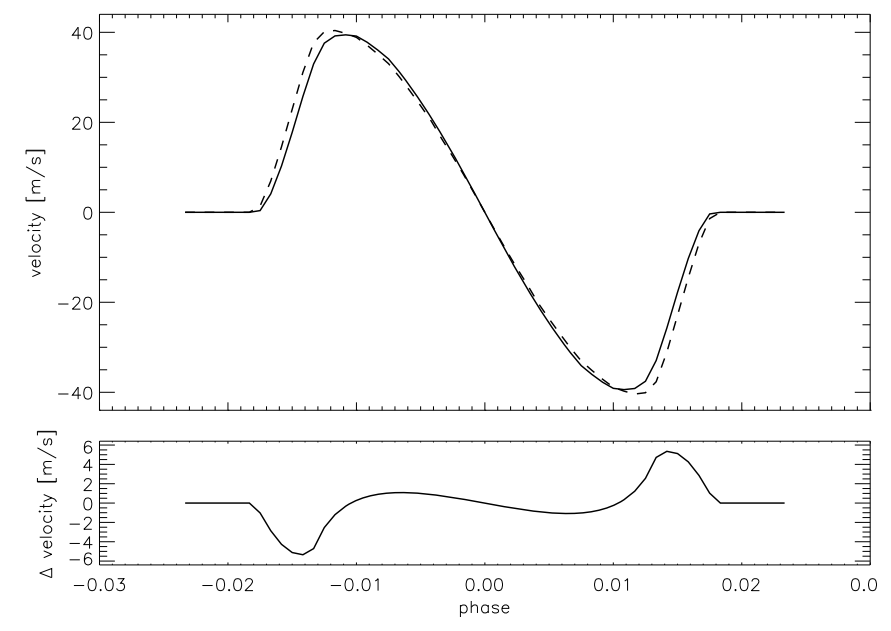

Fig. 3. Effect of limb-darkening: top panel: Rossiter-McLaughlin effect (orbital motion subtracted) for the wavelength region 5000-5200 from synthetic spectra using specific intensities (full line) compared to the application of a linear limb-darkening law (dashed line). Lower panel: difference between the full simulation and the limb-darkening approximation. See text for details.

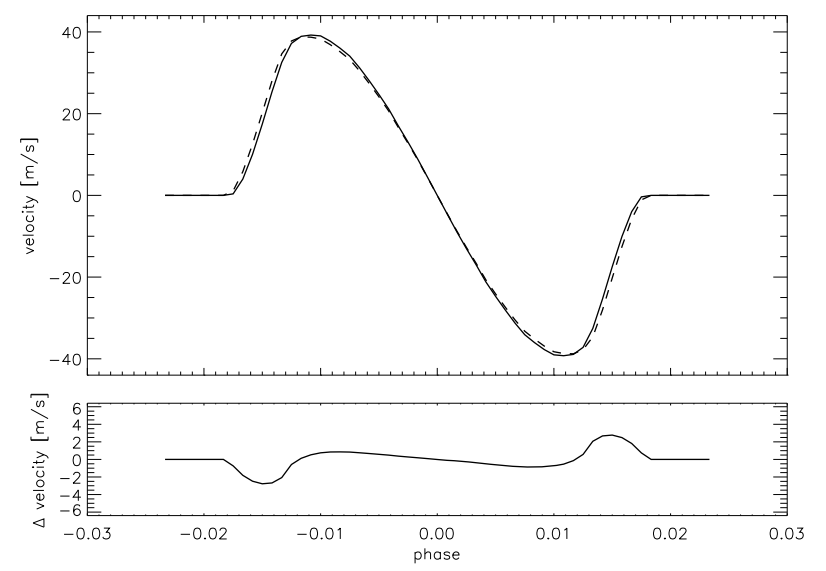

Fig. 4. As in Fig. 3, but for application of the wavelength-dependent linear limb-darkening law shown in Fig. 5.

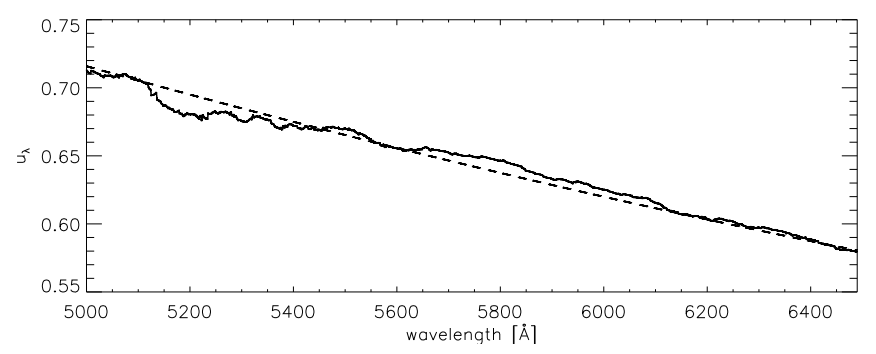

Fig. 5. Wavelength-dependent linear limb-darkening coefficient determined from the applied PHOENIX models smoothed over $100 \AA$ bins (full line) and polynomial fit of second order (dashed line).

of the specific intensities are greatest. A limb-darkening law therefore mocks a star with a different effective radius. The differences can be reduced with a wavelength-dependent limbdarkening (Fig. 4) law but not completely suppressed.
Systematic differences between the detailed treatment and a limb-darkening approximation are up to $6 \mathrm{~m} \mathrm{~s}^{-1}$ in the first case and $2 \mathrm{~m} \mathrm{~s}^{-1}$ in the second. A detailed treatment of the RME might therefore help to reduce the error bars in determinations of the spin-orbit alignments (or misalignements) of transiting extrasolar planets (Bundy \& Marcy 2000; Queloz et al. 2000; Winn et al. 2005, 2006, 2007; Narita et al. 2007; Wolf et al. 2007; Bouchy et al. 2008; Cochran et al. 2008; Hébrard et al. 2008; Johnson et al. 2008; Joshi et al. 2008; Loeillet et al. 2008; Narita et al. 2008). Because of the wavelength dependency, a detailed treatment also seems necessary in order to avoid confusion between effects of a wavelength-dependent planetary radius and a wavelength-dependent limb-darkening.

\subsection{The wavelength-dependent Rossiter-McLaughlin effect}

With our simulations and predictions for the wavelength dependency of planetary radii (Barman 2007; Fortney et al. 2008), it is now possible to simulate the wavelength dependency of the RME and to estimate the observability. All test calculations were applied to HD 209458. It should, however, be noted that our simulations are not restricted to that object. We assume a wavelength range from 5000-6500 $\AA$, roughly covered by a spectrograph stabilized by an iodine-cell and covering the NaDdoublet where the planetary radius is increased by about $5 \%$ due to absorption within the planetary atmosphere. We used the phase-dependent synthetic spectra as described in Sect. 2, performed cross correlations stepping through the spectral region with a step size of $25 \AA$, and a width of $100 \AA$, i.e. averaging the RME over each $100 \AA$-bins. The correspondingly averaged wavelength-dependent radius of HD 209458b can be seen in Fig. 1. As a reference, we use the RME averaged over the region 5000-5200 $\AA$, because the predicted radius is least influenced by absorption in the planetary atmosphere in our spectral region of interest. The differential effect is illustrated in Fig. 6, where we show the RME of the reference region, the $100 \AA$-bin centered around $\mathrm{NaD}$, as well as the difference between the two. The amplitude of the effect is about $1.5 \mathrm{~m} \mathrm{~s}^{-1}$, about the quantity derived by Snellen (2004). It should be noted that the differential effect is qualitatively different from the one caused by simplifications using parameterized limb-darkening laws (Figs. 3 and 4). The maximum effect occurs when the planet covers most of the blue- or red-shifted hemisphere, i.e. after the second and before the third contact. The wavelength dependency of the planetary radius therefore has a different signature in the RME than limbdarkening. Using a simplified limb-darkening model as comparison for the observations would in principle allow discrimination between effects of the limb-darkening and the wavelength dependency; in practice, the necessary accuracy would be difficult to achieve. A detailed model is therefore needed to obtain reliable results.

In Fig. 7 we show the wavelength dependency of these differences. The amplitude of the difference between the RME of each $100 \AA$-bin and the RME of the reference region are plotted against the central wavelength of each bin. While this indicates an signal of about $3 \mathrm{~m} \mathrm{~s}^{-1}$ with a peak at the $\mathrm{NaD}$ doublet, it should be noted that about half of the effect is due to the wavelength dependency of the limb-darkening, seen where we keep the planetary radius constant at $R_{\mathrm{P}}=1.31 R_{\text {jup }}$, the wavelength-dependent size corresponding to the reference region. Here we can also see a peak at $\mathrm{NaD}$, but with a lower amplitude $\left(1.5 \mathrm{~m} \mathrm{~s}^{-1}\right)$. The observations of Snellen (2004) 


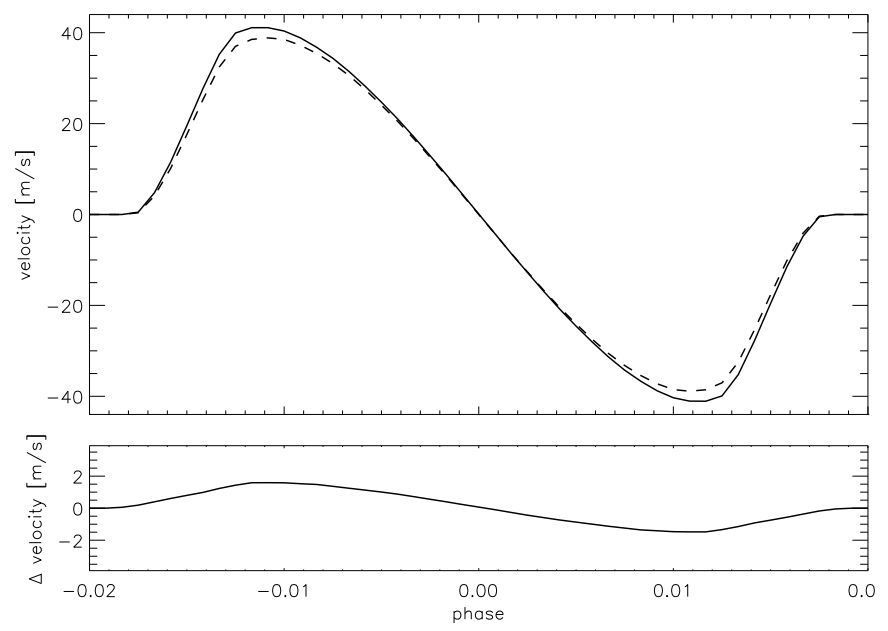

Fig. 6. Rossiter-McLaughlin effect (orbital motion subtracted) for the wavelength region 5840-5940 $\AA$ (100 $\AA$ bin centered on the NaDdoublet): simulation with the wavelength-dependent radius for that wavelength interval (top panel, full line) versus a fixed radius $R_{\mathrm{P}}=$ $1.31 R_{\text {jup }}$ (top panel, dashed line) and difference between the two (lower panel).
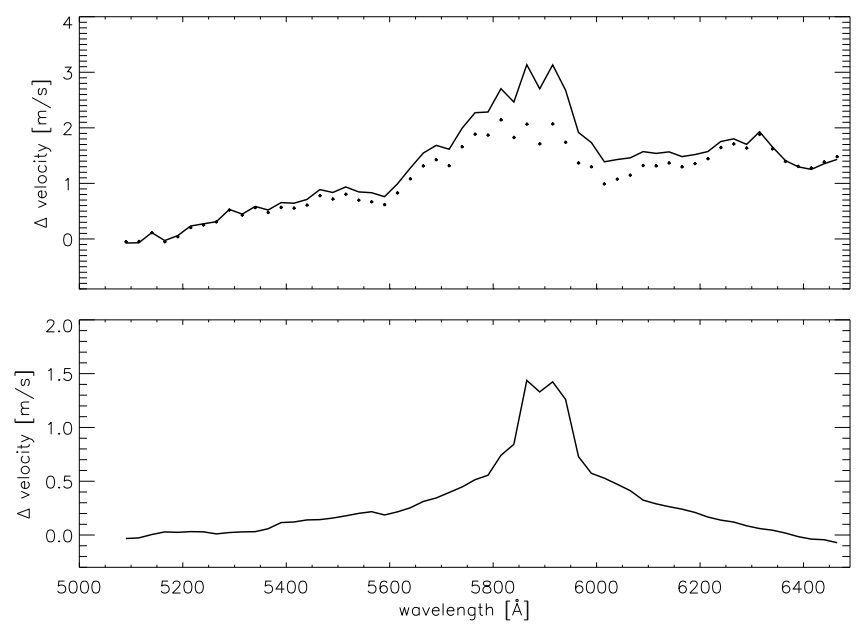

Fig. 7. Wavelength-dependent Rossiter-McLaughlin effect at the phase of maximum amplitude (-0.012): top panel: difference between the wavelength-dependent RME and the RME from the reference region 5000-5200 A. Full line: simulation with the wavelength-dependent radius; dotted line: simulations with a fixed radius $R_{\mathrm{P}}=1.31 R_{\text {jup }}$. Lower panel: the difference between the two simulations from the top panel.

indicate a measured amplitude of $1.7 \mathrm{~m} \mathrm{~s}^{-1}$ for HD 209458 from UVES spectra, in accordance to our simulations.

As a possible strategy for an analysis we therefore suggest using the observed RME of the reference region to obtain the necessary stellar and planetary parameters from a fit of the simulated RME (stellar radius, orientation of the orbit with respect to the rotation axis of the star, as well as with respect to the line of sight, and especially the planetary radius at this wavelengths) and use the corresponding synthetic RME as reference. The differential effect as displayed in the lower panels of Figs. 6 and 7 would then be the result of the analysis.

\subsection{The influence of the stellar rotation velocity}

For a broader application of these technique, the influence of the rotation velocity of the host star is the most critical parameter.

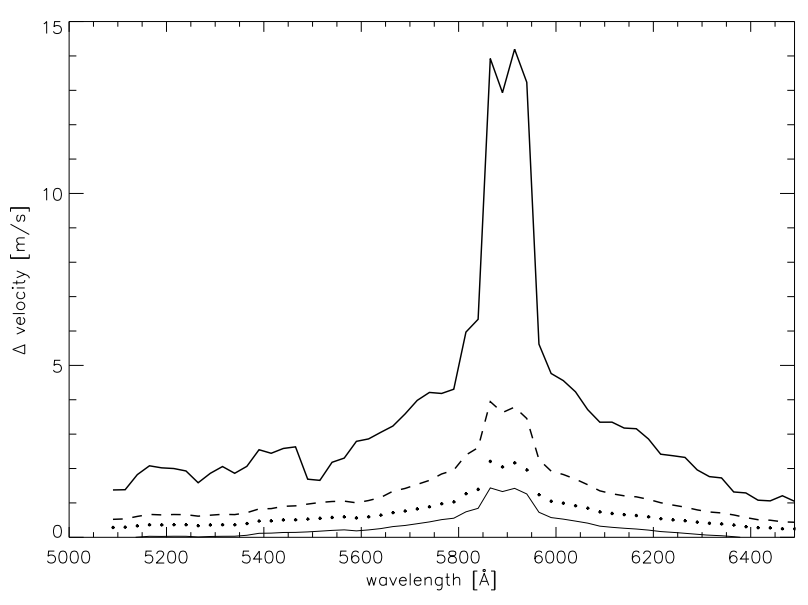

Fig. 8. The effect of stellar rotation: same as lower panel of Fig. 7 but for stellar rotation velocities of $v_{\mathrm{eq}}=4.5,6.0,9.0,25.0 \mathrm{~km} \mathrm{~s}^{-1}$ (thin, dotted, dashed, and thick lines).

While HD 209458 is a slow rotator, several of the known host stars of transiting planets rotate faster. To avoid confusion between the effects of changing stellar parameters for different host stars, we compared the results from the previous paragraph now to simulation for models where all parameters are kept fixed except the rotation velocity. The results are presented in Fig. 8. As expected, the amplitude of the observable effect increases with the rotation velocity of the host star. While the amplitude is about $1.5 \mathrm{~m} \mathrm{~s}^{-1}$ for a rotation velocity of $4.5 \mathrm{~km} \mathrm{~s}^{-1}$, it increases to about $4 \mathrm{~m} \mathrm{~s}^{-1}$ for a rotation velocity of $9 \mathrm{~km} \mathrm{~s}^{-1}$. For planet host stars with spectral types earlier than about F5, the rotation velocity is significantly larger compared to those of later spectral type. Bright planet host stars of earlier spectral types are therefore the primary targets of this method.

\subsection{Wavelength-dependent transit light curves}

As a by-product, we can also simulate transit light curves by integrating the flux for each phase point. We can therefore also provide detailed simulations for transmission photometry. As an example, we show in Fig. 9 the transit light curve for HD 209458 for the reference region 5000-5200 $\AA$ (assuming a box-like filter) and for the average of two $3 \AA$-bins, each centered on one of the NaD components. The difference between the two is provided in the lower panel as a possible observational result, similar to those of Charbonneau et al. (2002); Redfield et al. (2008); Snellen et al. (2008); Sing et al. (2008a). The differential effect is strongest during ingress and egress because the slightly larger planetary radius at the $\mathrm{NaD}$-lines causes a slightly early first and slightly later fourth contact. The differential effect during phases between the second and third contacts is a combination of the slightly deeper transit due to a larger radius at $\mathrm{NaD}$ and the differential effect of the limb-darkening between the two wavelength regions. This part of the curve therefore depends critically on the wavelengths of the reference region.

Since (ground-based) measurements of the differential effect of the transit light curves with wavelength regions of strong absorption lines of the planetary atmosphere rely on high-resolution and high-quality spectra, both the RME and the wavelength-dependent transit light curve can be evaluated simultaneously. With our simulations as comparison, the detection efficiency can therefore be increased significantly. 

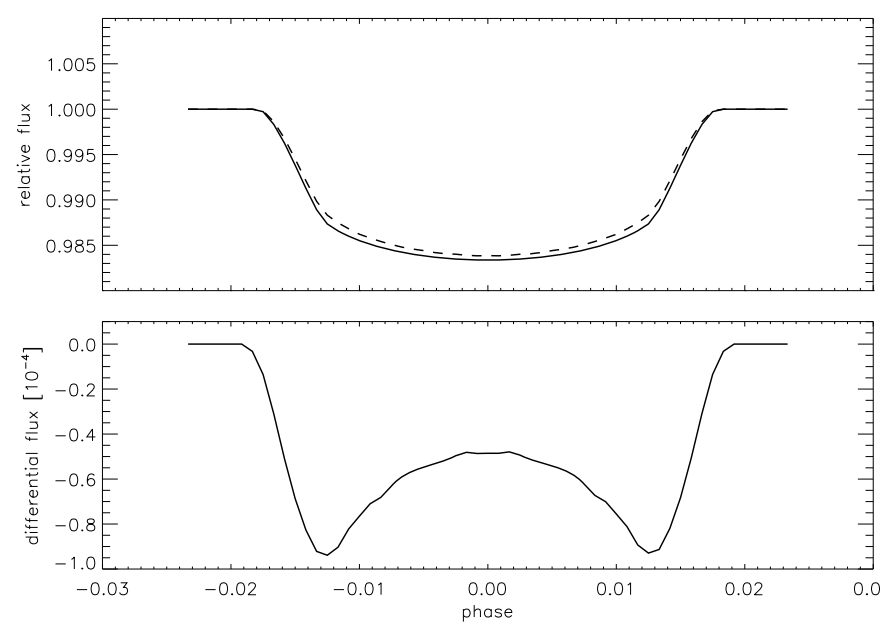

Fig. 9. Wavelength-dependent transit light curves: average from two wavelength regions centered on the two $\mathrm{NaD}$ doublet components of $3 \AA$ width each (top panel, full line), from a reference region 5000-5200 ̊ (dashed line), and the difference between the two (lower panel).

\section{Conclusion}

We performed simulations of the Rossiter-McLaughlin effect using a predicted wavelength dependency for the transiting planet in order to evaluate the possibility detecting atmospheres of extrasolar planets with ground-based high-resolution spectroscopy. We showed that a detailed treatment of the spectrum synthesis of the partly eclipsed stellar surface is important for detecting these subtle effects. In typical applications for the measurement of spin-orbit alignment, we obtained systematic differences up to $6 \mathrm{~m} \mathrm{~s}^{-1}$ in simulations tuned for HD 209458. A faster rotation of the primary star would increase these systematic effects.

The slightly larger planetary radius at the NaD-resonance line, caused by additional absorption within the planetary atmosphere, produces a slightly larger RME compared to a wavelength region where the planetary atmosphere makes a very small contribution (e.g. the region 5000-5200 A). Our simulations for HD 209458 predict an amplitude of the effect of $1.5 \mathrm{~m} \mathrm{~s}^{-1}$. This is a very small effect that requires several phaseresolved high-resolution spectra from several transits taken with a stabilized spectrograph. While the rotation velocity of HD 209458 is low $\left(4.5 \mathrm{~km} \mathrm{~s}^{-1}\right)$, systems with a primary of spectral type F5 or earlier typically show higher rotation velocities. For rotation velocities of $25 \mathrm{~km} \mathrm{~s}^{-1}$, the amplitude of the effect is already $15 \mathrm{~m} \mathrm{~s}^{-1}$. With increasing rotational velocity, the accuracy of the radial velocity measurements will, however, be reduced by the broadening of the stellar lines.

From these simulation, we can also derive wavelengthdependent transit light curves. A larger planetary radius at the $\mathrm{NaD}$-resonance line could be detected from comparing the light curve at this wavelength to one at a reference region with small contributions from the planetary atmosphere.

During recent years, atmospheres of extrasolar planets have been probed with different techniques. Photometric observations of the planetary albedo and secondary eclipses have to rely on space-based IR instruments, while the transit spectroscopy has recently been successful also from the ground (see Sect. 1). In this paper it has been been shown that the analysis of the wavelength-dependent Rossiter-McLaughlin effect is an alternative approach for a ground-based detection. While access from the ground will potentially enable broader application, the information of the ground-based transit spectroscopy as well as our proposed application of the RME, is restricted to strong absorption lines in the planetary atmosphere. An advantage of the wavelength-dependent RME is that it can be obtained as a byproduct of either ground-based transit spectroscopy, providing an independent detection of absorption lines in planetary atmospheres, or as by-product of RME measurements for spinorbit alignment, provided that the requirement for the wavelength stability of the spectrograph is high enough to measure the wavelength-dependent effect.

Acknowledgements. We thank P. Hauschildt for advice in computing the PHOENIX intensity spectra. A.R. acknowledges financial support through an Emmy Noether Fellowship from the Deutsche Forschungsgemeinschaft under DFG RE 1664/4-1.

\section{References}

Asplund, M., Grevesse, N., \& Sauval, A. J. 2005, in Cosmic Abundances as Records of Stellar Evolution and Nucleosynthesis, ed. T. G. Barnes, III, \& F. N. Bash, ASP Conf. Ser., 336, 25

Bakos, G. Á., Kovács, G., Torres, G., et al. 2007, ApJ, 670, 826

Barman, T. 2007, ApJ, 661, L191

Bouchy, F., Queloz, D., Deleuil, M., et al. 2008, A\&A, 482, L25

Brott, I., \& Hauschildt, P. H. 2005, in The Three-Dimensional Universe with Gaia, ed. C. Turon, K. S. O'Flaherty, \& M. A. C. Perryman, ESA Special Publication, 576, 565

Brown, T. M., Charbonneau, D., Gilliland, R. L., Noyes, R. W., \& Burrows, A. 2001, ApJ, 552, 699

Bundy, K. A., \& Marcy, G. W. 2000, PASP, 112, 1421

Charbonneau, D., Brown, T. M., Noyes, R. W., \& Gilliland, R. L. 2002, ApJ, 568,377

Charbonneau, D., Allen, L. E., Megeath, S. T., et al. 2005, ApJ, 626, 523

Claret, A. 2000, A\&A, 363, 1081

Claret, A. 2003, A\&A, 401, 657

Claret, A. 2004, A\&A, 428, 1001

Cochran, W. D., Redfield, S., Endl, M., \& Cochran, A. L. 2008, ApJ, 683, L59

Deming, D., Seager, S., Richardson, L. J., \& Harrington, J. 2005, Nature, 434, 740

Deming, D., Harrington, J., Seager, S., \& Richardson, L. J. 2006, ApJ, 644, 560

Deming, D., Harrington, J., Laughlin, G., et al. 2007, ApJ, 667, L199

Demory, B.-O., Gillon, M., Barman, T., et al. 2007, A\&A, 475, 1125

Ehrenreich, D., Lecavelier Des Etangs, A., Hébrard, G., et al. 2008, A\&A, 483, 933

Fischer, D. A., Vogt, S. S., Marcy, G. W., et al. 2007, ApJ, 669, 1336

Fortney, J. J., Lodders, K., Marley, M. S., \& Freedman, R. S. 2008, ApJ, 678, 1419

Giménez, A. 2006, ApJ, 650, 408

Grillmair, C. J., Charbonneau, D., Burrows, A., et al. 2007, ApJ, 658, L115

Harrington, J., Luszcz, S., Seager, S., Deming, D., \& Richardson, L. J. 2007, Nature, 447, 691

Hauschildt, P. H., \& Baron, E. 1999, J. Comput. Appl. Math., 109, 41

Hauschildt, P. H., Allard, F., Ferguson, J., Baron, E., \& Alexander, D. R. 1999, ApJ, 525, 871

Hébrard, G., Bouchy, F., Pont, F., et al. 2008, A\&A, 488, 763

Hosokawa, Y. 1953, PASJ, 5, 88

Johns-Krull, C. M., McCullough, P. R., Burke, C. J., et al. 2008, ApJ, 677, 657

Johnson, J. A., Winn, J. N., Narita, N., et al. 2008, ApJ, 686, 649

Joshi, Y. C., Pollacco, D., Collier Cameron, A., et al. 2008, ArXiv e-prints

Kopal, Z. 1959, Close binary systems (The International Astrophysics Series London: Chapman \& Hall)

Lecavelier Des Etangs, A., Pont, F., Vidal-Madjar, A., \& Sing, D. 2008a, A\&A, 481, L83

Lecavelier Des Etangs, A., Vidal-Madjar, A., Désert, J.-M., \& Sing, D. 2008b, A\&A, 485, 865

Loeillet, B., Shporer, A., Bouchy, F., et al. 2008, A\&A, 481, 529

McLaughlin, D. B. 1924, ApJ, 60, 22

Narita, N., Enya, K., Sato, B., et al. 2007, PASJ, 59, 763

Narita, N., Sato, B., Ohshima, O., \& Winn, J. N. 2008, PASJ, 60, L1

Nordström, B., Mayor, M., Andersen, J., et al. 2004, A\&A, 418, 989

Ohta, Y., Taruya, A., \& Suto, Y. 2005, ApJ, 622, 1118

Petrie, R. M. 1938, Publications of the Dominion Astrophysical Observatory Victoria, 7, 105

Pont, F., Knutson, H., Gilliland, R. L., Moutou, C., \& Charbonneau, D. 2008, MNRAS, 385, 109 
Queloz, D., Eggenberger, A., Mayor, M., et al. 2000, A\&A, 359, L13

Redfield, S., Endl, M., Cochran, W. D., \& Koesterke, L. 2008, ApJ, 673, L87

Richardson, L. J., Deming, D., Horning, K., Seager, S., \& Harrington, J. 2007, Nature, 445, 892

Rossiter, R. A. 1924, ApJ, 60, 15

Schlesinger, F. 1910, Publications of the Allegheny Observatory of the University of Pittsburgh, 1, 123

Sing, D. K., Vidal-Madjar, A., Désert, J.-M., Lecavelier des Etangs, A., \& Ballester, G. 2008a, ApJ, 686, 658

Sing, D. K., Vidal-Madjar, A., Lecavelier des Etangs, A., et al. 2008b, ApJ, 686, 667

Snellen, I. A. G. 2004, MNRAS, 353, L1

Snellen, I. A. G., Albrecht, S., de Mooij, E. J. W., \& Le Poole, R. S. 2008, A\&A, 487,357
Swain, M. R., Bouwman, J., Akeson, R. L., Lawler, S., \& Beichman, C. A. 2008a, ApJ, 674, 482

Swain, M. R., Vasisht, G., \& Tinetti, G. 2008b, Nature, 452, 329

Tinetti, G., Vidal-Madjar, A., Liang, M.-C., et al. 2007, Nature, 448, 169

Townsend, R. H. D. 1997, MNRAS, 284, 839

Vidal-Madjar, A., Lecavelier des Etangs, A., Désert, J.-M., et al. 2003, Nature, 422,143

Vidal-Madjar, A., Désert, J.-M., Lecavelier des Etangs, A., et al. 2004, ApJ, 604, L69

Winn, J. N., Noyes, R. W., Holman, M. J., et al. 2005, ApJ, 631, 1215

Winn, J. N., Johnson, J. A., Marcy, G. W., et al. 2006, ApJ, 653, L69

Winn, J. N., Johnson, J. A., Peek, K. M. G., et al. 2007, ApJ, 665, L167

Wolf, A. S., Laughlin, G., Henry, G. W., et al. 2007, ApJ, 667, 549 